RAD HAZU. MATEMATIČKE ZNANOSTI

Vol. $23=538$ (2019): 107-122

DOI: https://doi.org/10.21857/y54jofpplm

\title{
MINDING ISOMETRIES OF RULED SURFACES IN LORENTZ-MINKOWSKI SPACE
}

\author{
Ljiljana Primorac Gajčić and Želuka Milin Šipuš
}

\begin{abstract}
In this paper we study isometries of ruled surfaces in the Lorentz-Minkowski space that preserve rulings. A special attention is given to the classes of surfaces having no Euclidean counterparts. We also construct some examples of isometric ruled surfaces with certain properties and rulings preserved.
\end{abstract}

\section{INTRODUCTION}

A classical topic in Euclidean differential geometry is the study of local isometries between surfaces. Local isometries, i.e. differentiable mappings between surfaces that preserve their first fundamental forms (or intrinsic metrics), preserve their Gaussian curvatures, but do not determine a surface uniquely (up to a space isometry). The classical example is the existence of a local isometry between a right helicoid and a catenoid. It is of particular interest to study local isometries by adding certain additional conditions or to study isometries between surfaces of some special classes. There are results that state how much geometric information of a surface is sufficient to determine a surface uniquely. Such information is provided by e.g. the first and the second fundamental form of a surface, but not by its first fundamental form and the principal curvatures. Surfaces having the same the first fundamental forms and the principal curvatures are called the Bonnet surfaces. They can be defined equivalently as isometric surfaces having the same mean curvatures. The classical Euclidean result states that any surface of constant mean curvature admits a one-parameter family of isometric deformation. Since right helicoid and catenoid are both minimal, local isometry between them is obtained through associated family of minimal surfaces. On the other side, the Bour's theorem states that there exist local isometries between helicoidal and rotation surfaces so that helices on the helicoidal surface correspond to circles on the rotation surfaces. Further, consideration of local

2010 Mathematics Subject Classification. 53A35, 53B30, 53C50.

Key words and phrases. Lorentz-Minkowski space, isometry, ruled surface, $B$-scroll. 
isometries in the class of ruled surfaces naturally imposes a new question on conditions for local isometry to preserve rulings of ruled surfaces. Existence of an isometry between a helicoid (a ruled surface) and a catenoid (a rotation surface) shows that a condition for the image surface to be ruled is not trivial. The less known result states that if two ruled surfaces are locally isometric, then the local isometry preserves their rulings, unless the surfaces are isometric to a doubly-ruled quadric surface whose rulings correspond to the rulings of both initial ruled surfaces, [5]. This result also gives the motivation to study isometries between ruled surfaces that preserve their rulings. In Euclidean space these mappings were first studied by F. Minding and therefore called the Minding isometries. A differential geometric treatment of that problem goes back to Kruppa, [14].

In this paper we analyze the analogous problem in 3-dimensional LorentzMinkowski space. The Lorentz-Minkowski space usually provides a richer context than the Euclidean space since the defined pseudo-metric offers more variations in geometrical notions, [15]. We give a special attention to the classes of surfaces which have no Euclidean counterparts. Different topics of the classical differential geometry of surfaces in the Lorentz-Minkowski space can be found in many papers. Ruled surfaces have been studied in e.g. [1-3,9,11-13]. The analogue of the Bour's theorem in the Lorentz-Minkowski space can be found in [8], and the Bonnet surfaces in [7]. In [16] the so called BonnetThompsen spacelike surfaces are studied, i.e. spacelike surfaces that are both maximal and affine minimal. The Minding isometries of ruled surfaces in a simply isotropic space $I_{3}^{1}$ are studied in [17] and in pseudo-Galilean space in $[4]$.

\section{Preliminaries}

The Lorentz-Minkowski 3-space $\mathbb{R}_{1}^{3}$ is a pseudo-Euclidean space endowed with a non-degenerate indefinite symmetric bilinear form of index 1 (a pseudoscalar product) defined by

$$
\langle x, y\rangle=x_{1} y_{1}+x_{2} y_{2}-x_{3} y_{3}
$$

for $x=\left(x_{1}, x_{2}, x_{3}\right)$ and $y=\left(y_{1}, y_{2}, y_{3}\right)$. A vector $x \in \mathbb{R}_{1}^{3}$ can have one of three causal character: it is spacelike if $\langle x, x\rangle>0$ or $x=0$, timelike if $\langle x, x\rangle<0$ and lightlike (null, isotropic) if $\langle x, x\rangle=0$ and $x \neq 0$. The pseudo-norm of a vector $x$ is defined as the real number $\|x\|=\sqrt{|\langle x, x\rangle|} \geq 0$. Similarly, the causal character of an arbitrary curve $\alpha=\alpha(s) \in \mathbb{R}_{1}^{3}$ is determined by the causal character of its velocity vector $\alpha^{\prime}(s)$.

Let $\mathbb{S}$ be a smooth immersed surface in $\mathbb{R}_{1}^{3}$ with a local parametrization $f: U \rightarrow \mathbb{R}_{1}^{3}$, where $U \subset \mathbb{R}^{2}$ is an open set. The causal character of a surface $\mathbb{S}$ is determined by its first fundamental form, i.e. a surface $\mathbb{S}$ is called spacelike (resp. timelike, null or lightlike) if its first fundamental form $g$ is positive 
definite (resp. indefinite, of rank 1). From now on we consider only non-null surfaces. For spacelike (resp. timelike) surfaces we define locally the unit normal field

$$
n=\frac{f_{1} \times_{L} f_{2}}{\left\|f_{1} \times_{L} f_{2}\right\|},
$$

which is a timelike (resp. spacelike) field. For any two vectors $v=\left(v_{1}, v_{2}, v_{3}\right)$ and $w=\left(w_{1}, w_{2}, w_{3}\right) \in \mathbb{R}_{1}^{3}$, the Lorentzian cross-product $v \times_{L} w$ of $v$ and $w$ is defined as $v \times{ }_{L} w=J(v \times w)$, where $\times$ is the Euclidean cross-product and

$$
J=\left(\begin{array}{ccc}
1 & 0 & 0 \\
0 & 1 & 0 \\
0 & 0 & -1
\end{array}\right) .
$$

The shape operator of a surface $\mathbb{S}$ (at point $p$ derived from $n$ ) is a self-adjoint operator with respect to $\langle\rangle,,\left\langle L_{p} v, w\right\rangle=\left\langle v, L_{p} w\right\rangle, v, w \in T_{p} \mathbb{S}$. The eigenvalues $k_{1}, k_{2}$ of the shape operator $L_{p}$ (real or complex conjugate) are called the principal curvatures of $\mathbb{S}$. When they are real, the associated eigenvectors are the principal directions of a surface $\mathbb{S}$ in $p$. There are always two principal curvatures on the spacelike part of $\mathbb{S}$ and the shape operator is diagonalizable. In the case of timelike surfaces, eigenvalues of $L_{p}$ can either be real and different ( $L_{p}$ is diagonalizable), real and equal ( $L_{p}$ need not be diagonalizable) or complex conjugate ( $L_{p}$ is diagonalizable). The Gaussian curvature of $\mathbb{S}$ is in local coordinates given by

$$
K=\epsilon \frac{L N-M^{2}}{E G-F^{2}}
$$

whereas for the mean curvature we have

$$
H=\frac{\epsilon}{2} \frac{L G-2 F M+E N}{E G-F^{2}},
$$

where $E, F$ and $G$ are coefficients of the first fundamental form, $L, M$ and $N$ are coefficients of the second fundamental form and $\epsilon=\langle n, n\rangle$.

\section{Minding isometries of RUled SURfaces in $\mathbb{R}_{1}^{3}$}

A ruled surface $\mathbb{S}$ in $\mathbb{R}_{1}^{3}$ is a surface traced out by a straight line moving along curve $c$ and therefore it admits a local parametrization $\mathbf{x}: I \times \mathbb{R} \rightarrow \mathbb{R}_{1}^{3}$ of the form

$$
\mathbf{x}(u, v)=c(u)+v r(u) .
$$

The curve $c$ is the base curve (the generating curve) and the straight lines with directions $r(u)$ are the rulings of a surface.

In this paper we consider all three classes of ruled surfaces in $\mathbb{R}_{1}^{3}$. According to [1], [3], there are three main classes of ruled surfaces in $\mathbb{R}_{1}^{3}$ : surfaces having rulings $r$ and their derivative $r^{\prime}$ nowhere null (the class $M_{1}^{1}$ ), surfaces having $r$ nowhere null but $r^{\prime}$ null everywhere (the class $M_{1}^{0}$ ) and surfaces with 
$r$ null everywhere (the class $M_{0}$ ). While the surfaces of the class $M_{1}^{1}$ are analogues of Euclidean ruled surfaces, surfaces of the class $M_{1}^{0}$, respectively $M_{0}$ have no Euclidean counterparts. Here we study Minding isometries for every class separately with particular interest to analyze local isometries for surfaces of class $M_{1}^{0}$ and special type of surfaces of class $M_{0}$, so-called $B$-scrolls [10]. We show that surfaces of class $M_{1}^{0}$ admit only trivial isometry and describe Minding isometries for other two classes of surfaces.

The surfaces with $r$ and $r^{\prime}$ nowhere null will be referred as non-null ruled surface, while those with $r$ null everywhere will be called null-ruled surfaces. Among non-null ruled surfaces we distinguish three types of surfaces with respect to the causal character of the base curve, respectively of the rulings of a ruled surface. A non-null ruled surface is called of type $\mathbb{S}_{1}$, (resp. $\mathbb{S}_{2}$, $\mathbb{S}_{3}$ ) if its base curve is spacelike (resp. spacelike, timelike) curve and rulings are spacelike (resp. timelike, spacelike) vectors. A null-ruled surface can be parametrized as a $B$-scroll, i.e. timelike ruled surface with a base curve which is a null Frenet curve with a null frame $(A, B, C)$ and null rulings determined by the field $B,[10]$.

Now, let $\mathbb{S}, \overline{\mathbb{S}} \in \mathbb{R}_{1}^{3}$ be two surfaces given by their parameterizations $f: I \times \mathbb{R} \rightarrow \mathbb{R}_{1}^{3}, \bar{f}: \bar{I} \times \mathbb{R} \rightarrow \mathbb{R}_{1}^{3}$, respectively. Let $F: \mathbb{S} \rightarrow \overline{\mathbb{S}}$ be a mapping of the surfaces, $F_{* p}: T_{p} \mathbb{S} \rightarrow T_{F(p)} \overline{\mathbb{S}}$, its differential (the push-forward). Then $F$ is called a local isometry if $F_{* p}$ preserves the pseudo-scalar product, i.e. $\left\langle F_{* p}(v), F_{* p}(w)\right\rangle=\langle v, w\rangle, p \in \mathbb{S}, v, w \in T_{p} \mathbb{S}$.

We will call a mapping $F: \mathbb{S} \rightarrow \overline{\mathbb{S}}$ the Minding isometry if $F$ is a local isometry which maps the rulings of $\mathbb{S}$ into the rulings of $\overline{\mathbb{S}}$.

Note that a local isometry preserves the causal character of a vector (respectively curve, surface), therefore we can define Minding isometry only between surfaces whose rulings have the same causal character. Furthermore, it will be shown that we can consider Minding isometries only between ruled surfaces with base curves of the same causal character.

\section{Minding isometries OF NON-NUll RULed SURfaCeS}

Let $\mathbb{S}$ be a surface of a class $M_{1}^{1}$ with parametrization (3.1). If consecutive rulings of a ruled surface in $\mathbb{R}_{1}^{3}$ intersect or are parallel, then the surface is said to be developable. These surfaces can be mapped isometrically into the plane. All other ruled surfaces are called skew surfaces. The striction point on a ruled surface is the foot of the common perpendicular in the sense of the metric of $\mathbb{R}_{1}^{3}$ to two consecutive rulings. The set of the striction points defines a curve on a ruled surface $\mathbb{S}$ called the striction curve, whose parametrization is given by

$$
s(u)=c(u)-\frac{\left\langle c^{\prime}(u), r^{\prime}(u)\right\rangle}{\left\langle r^{\prime}(u), r^{\prime}(u)\right\rangle} r(u)
$$


In particular, a skew non-null ruled surface $\mathbb{S} \in \mathbb{R}_{1}^{3}$ with parametrization (3.1) always admits the reparameterization

$$
\tilde{\mathbf{x}}(u, v)=s(u)+v e(u),
$$

where $e(u)=\frac{r(u)}{\|r(u)\|}, e^{2}= \pm 1$, and $s(u)$ is the striction curve of such a surface, that is $\left\langle s^{\prime}(u), e^{\prime}(u)\right\rangle=0$ holds.

The distribution parameter of the ruled surface with parametrization (4.2) is defined as

$$
D=\frac{\operatorname{det}\left(s^{\prime}, e, e^{\prime}\right)}{\left\langle e^{\prime}, e^{\prime}\right\rangle} .
$$

If $D=0$, then normal vectors are collinear along the same ruling at nonsingular points of the surface, i.e. the tangent planes are identical. These surfaces are developable. Skew ruled surfaces are characterized by the distribution parameter $D \neq 0$.

Without loss of generality, we can assume that considered ruled surfaces have parametrization (4.2) where $u$ is arc length parameter of its striction curve. Analogously as in the Euclidean case, non-null ruled surfaces are described, up to Lorentzian motion, by the analogue of the moving Sannia frame, which provides a complete system of invariants of ruled surface, the curvature $\kappa$, the torsion $\tau$ and the striction $\sigma$, [6,19]. For a ruled surface $\mathbb{S}_{1}$ with parametrization (4.2) by choosing $n=\frac{e^{\prime}}{\left\|e^{\prime}\right\|}$ and $z=e \times n$, we obtain orthonormal frame $\{e, n, z\}$, where $n$ is timelike and $e$ and $z$ are spacelike vectors. The Frenet formulas of this frame along $e$ become

$$
e^{\prime}=\kappa n, \quad n^{\prime}=\kappa e+\tau z, \quad z^{\prime}=\tau n,
$$

where $\kappa$ and $\tau$ are referred as curvature and torsion, respectively, at the ruling $e(u)$. The tangent vector of a striction curve of $\mathbb{S}_{1}$ is a spacelike vector $s^{\prime}=\cos \sigma e+\sin \sigma z$, where the angle $\sigma$, an angle enclosed by $s^{\prime}$ and $e$, is its striction. Hence, the spacelike ruled surface $\mathbb{S}_{1}$ can be parameterized by

$$
\mathbf{x}(u, v)=\int(\cos \sigma e(u)+\sin \sigma z(u)) \mathrm{d} u+v e(u) .
$$

Similarly, for a ruled surface $\mathbb{S}_{2}$, with orthonormal frame $\{e, n, z\}$, where $e$ is timelike and $n$ and $z$ are spacelike vectors the Frenet formulas are expressed by

$$
e^{\prime}=\kappa n, n^{\prime}=\kappa e-\tau z, z^{\prime}=\tau n .
$$

For a ruled surface $\mathbb{S}_{3}$, with orthonormal frame $\{e, n, z\}$, where $z$ is timelike and $e$ and $n$ are spacelike vectors the Frenet formulas are expressed by

$$
e^{\prime}=\kappa n, n^{\prime}=-\kappa e+\tau z, z^{\prime}=\tau n .
$$

Since for both of these surfaces, a tangent vector stays in the timelike plane determined by $e$ and $z$, it is given by $s^{\prime}=\sinh \sigma e+\cosh \sigma z$, where the real 
number $\sigma$, a Lorentzian timelike angle enclosed by $s^{\prime}$ and $e$, is its striction. Hence, both of these surfaces can be parameterized by

$$
x(u, v)=\int(\sinh \sigma e(u)+\cosh \sigma z(u)) \mathrm{d} u+v e(u) .
$$

The first fundamental forms $I=E \mathrm{~d} u^{2}+2 F \mathrm{~d} u \mathrm{~d} v+G \mathrm{~d} v^{2}$ of considered ruled surfaces $\mathbb{S}_{1}, \mathbb{S}_{2}, \mathbb{S}_{3}$, are given by:

- for a spacelike ruled surface $\mathbb{S}_{1}$

$$
I=\left(1-v^{2} \kappa^{2}\right) \mathrm{d} u^{2}+2 \cos \sigma \mathrm{d} u \mathrm{~d} v+\mathrm{d} v^{2},
$$

- for a timelike ruled surface $\mathbb{S}_{2}$

$$
I=\left(1+v^{2} \kappa^{2}\right) \mathrm{d} u^{2}-2 \sinh \sigma \mathrm{d} u \mathrm{~d} v-\mathrm{d} v^{2},
$$

- for a timelike ruled surface $\mathbb{S}_{3}$

$$
I=\left(-1+v^{2} \kappa^{2}\right) \mathrm{d} u^{2}+2 \sinh \sigma \mathrm{d} u \mathrm{~d} v+\mathrm{d} v^{2} .
$$

Also, the second fundamental forms $I I=L \mathrm{~d} u^{2}+2 M \mathrm{~d} u \mathrm{~d} v+N \mathrm{~d} v^{2}$ are given by:

- for a spacelike ruled surface $\mathbb{S}_{1}$

$$
\begin{aligned}
I I & =\frac{\kappa \cos \sigma \sin \sigma+\tau \sin ^{2} \sigma+v \kappa^{\prime} \sin \sigma-\sigma^{\prime} \cos \sigma v \kappa+v^{2} \kappa^{2} \tau}{\sqrt{\left|-\sin ^{2} \sigma+v^{2} \kappa^{2}\right|}} \mathrm{d} u^{2} \\
& +\frac{\kappa \sin \sigma}{\sqrt{\left|-\sin ^{2} \sigma+v^{2} \kappa^{2}\right|}} \mathrm{d} u \mathrm{~d} v,
\end{aligned}
$$

- for a timelike ruled surface $\mathbb{S}_{2}$

$$
\begin{aligned}
I I & =\frac{\kappa \sinh \sigma \cosh \sigma+\tau \cosh ^{2} \sigma+v \kappa^{\prime} \cosh \sigma+\sigma^{\prime} \sinh \sigma v \kappa-v^{2} \kappa^{2} \tau}{\sqrt{\left|\cosh ^{2} \sigma+v^{2} \kappa^{2}\right|}} \mathrm{d} u^{2} \\
& +\frac{\kappa \cosh \sigma}{\sqrt{\left|\cosh ^{2} \sigma+v^{2} \kappa^{2}\right|}} \mathrm{d} u \mathrm{~d} v,
\end{aligned}
$$

- for a timelike ruled surface $\mathbb{S}_{3}$

$$
\begin{aligned}
I I & =\frac{\kappa \sinh \sigma \cosh \sigma+\tau \cosh ^{2} \sigma+v \kappa^{\prime} \cosh \sigma-\sigma^{\prime} \sinh \sigma v \kappa-v^{2} \kappa^{2} \tau}{\sqrt{\left|\cosh ^{2} \sigma-v^{2} \kappa^{2}\right|}} \mathrm{d} u^{2} \\
& +\frac{\kappa \cosh \sigma}{\sqrt{\left|\cosh ^{2} \sigma-v^{2} \kappa^{2}\right|}} \mathrm{d} u \mathrm{~d} v .
\end{aligned}
$$

We can notice that for all three types of considered ruled surfaces, $I$ does not depend on the torsion and does not react to the change of sign of curvature $\kappa$ and for surfaces $\mathbb{S}_{1}$ also does not react to the change of sign of striction $\sigma$.

Furthermore, the rulings of the surfaces are preserved by the isometry $F: \mathbb{S} \rightarrow \overline{\mathbb{S}}$ if the coordinate mapping $(u, v) \mapsto(\bar{u}, \bar{v})$ is of the form $\bar{u}=f(u)$, 
$\bar{v}=v+g(u)$. After applying the condition that the first fundamental forms are preserved, by a simple calculation we obtain $\bar{u}=u, \bar{v}=v$.

Proposition 4.1. A striction curve of a surface $\mathbb{S}$ is mapped by the Minding isometry into a striction curve of a surface $\overline{\mathbb{S}}$.

Proof. The statement follows from $v=\bar{v}$.

Hence, for all three types of considered ruled surfaces the Minding isometries are described by the change of the sign of the function $\kappa$ and replacement of $C^{1}$-function $\tau: I \rightarrow \mathbb{R}$ by an arbitrary $C^{1}$-function $\bar{\tau}: \bar{I} \rightarrow \mathbb{R}$, and for the surfaces $\mathbb{S}_{1}$ also by the change of the sign of the function $\sigma$. Furthermore, the following proposition holds

Proposition 4.2. The absolute value $|D|$ of parameter of distribution of a ruled surface $\mathbb{S}_{1}(\kappa, \tau, \sigma)$ (resp. $\left.\mathbb{S}_{2}, \mathbb{S}_{3}\right)$ is invariant under Minding isometries.

Proof. From (4.3) it follows that $D=\frac{\sin \sigma}{\kappa}$, respectively, $D=\frac{\cosh \sigma}{\kappa}$, depending on the type of the surface. This is obviously invariant under Minding isometries.

The following problems involve determination of torsion of a ruled surface for all three types of non-null ruled surfaces, respecting that a ruled surface can be mapped by Minding isometry only to a ruled surface of the same type and are proved analogously as in the Euclidean case.

THEOREM 4.3. A ruled surface $\mathbb{S}$ can be mapped by the Minding isometry into a surface $\overline{\mathbb{S}}$ in the way that:

1. the surface $\overline{\mathbb{S}}$ is conoidal $(\bar{\tau}=0)$;

2. the surface $\overline{\mathbb{S}}$ is a surface of constant slope $\left(\frac{\overline{\bar{\tau}}}{\overline{\bar{\kappa}}}=\right.$ const.);

3. a given curve $v=v(u)$ on $\mathbb{S}$ is mapped into an asymptotic curve of $\overline{\mathbb{S}}$;

4. a given curve $v=v(u)$ on $\mathbb{S}$ is mapped into a line of curvature of $\overline{\mathbb{S}}$;

5. a given geodesic on $\mathbb{S}$ is mapped into a straight line of $\overline{\mathbb{S}}$;

6. a given curve $v=v(u)$ on $\mathbb{S}$ is mapped into a plane curve on $\overline{\mathbb{S}}$;

7. a given curve $v=v(u)$ on $\mathbb{S}$ is mapped into a curve on $\overline{\mathbb{S}}$ along which the mean curvature $\bar{H}$ of $\overline{\mathbb{S}}$ vanishes.

\section{LOCAL ISOMETRIES OF RULED SURFACES OF CLASS $M_{1}^{0}$}

Ruled surfaces with rulings $r$ nowhere null, but $r^{\prime}$ null everywhere are special class of ruled surfaces in $\mathbb{R}_{1}^{3}$, having no Euclidean counterparts. These surfaces were studied in [1]-[3]. Let $\mathbb{S}$ be such surface with parametrization (3.1). Notice since $r^{\prime}$ is a null vector, these surfaces do not admit reparametrization of the form (4.2) and therefore can not be described with analogue of the Sannia frame. According to [3], these surfaces are described, up to Lorentzian 
motion, with invariants $Q$ and $R$ defined by $Q:=\operatorname{det}\left(c^{\prime}, r, r^{\prime}\right), R:=\left\langle r^{\prime}, r^{\prime}\right\rangle$, respectively. Their first fundamental form is given by

$$
I=(R+2 Q v) \mathrm{d} u^{2}+\mathrm{d} v^{2} .
$$

Since $I$ depends on invariants $Q$ and $R$ which completely determine the ruled surface, we can conclude following

TheOREM 5.1. Two ruled surfaces of class $M_{1}^{0}$ are locally isometric if and only if they are congruent.

\section{Minding ISOMETRIES OF $B$-SCROLLS}

Every null scroll, i.e. null-rulled surface, satisfying $\left\langle c^{\prime}, r\right\rangle \neq 0$ admits a parametrization as a $B$-scroll, i.e. as a ruled surface with null rulings and with a base curve $c$ which is a null Frenet curve with a null frame $(A, B, C)$,

$$
\begin{gathered}
c^{\prime}=A, \quad A^{2}=B^{2}=0, \quad C^{2}=1, \\
\langle A, B\rangle=1,\langle A, C\rangle=0,\langle B, C\rangle=0 .
\end{gathered}
$$

Rulings are determined by the field $r=B$. Therefore, a $B$-scroll is parametrized by

$$
f(u, v)=c(u)+v B(u), u \in I \subset \mathbf{R}, v \in \mathbf{R} .
$$

Vector fields $(A, B, C)$ satisfy analogous formulas to Frenet formulas

$$
A^{\prime}=k_{2} C, B^{\prime}=k_{3} C, C^{\prime}=-k_{3} A-k_{2} B,
$$

where functions $k_{2}, k_{3}$ are called the curvatures of $c$. $B$-scrolls are timelike surfaces with the first fundamental form

$$
I=k_{3}^{2} v^{2} \mathrm{~d} u^{2}+2 \mathrm{~d} u \mathrm{~d} v
$$

the second fundamental form

$$
I I=\left(k_{2}+v k_{3}^{\prime}+v^{2} k_{3}^{3}\right) \mathrm{d} u^{2}+2 k_{3} \mathrm{~d} u \mathrm{~d} v
$$

and the shape-operator in a point $\mathrm{p} L_{p}$ given by (see [10])

$$
L_{p}=\left(\begin{array}{cc}
k_{3} & 0 \\
k_{2}+v k_{3}^{\prime} & k_{3}
\end{array}\right) .
$$

The Gaussian curvature $K$ and the mean curvature $H$ of $B$-scrolls are given by

$$
K=k_{3}^{2}, \quad H=k_{3} .
$$

We also consider $B$-scrolls of constant slope. These surfaces are characterized by $k_{2}=0$ or $k_{2} \neq 0$ and $\frac{k_{3}}{k_{2}}=$ const., [18]. Contrary to the case of Euclidean space, there are no conoidal surfaces among non-cylindrical null-ruled surfaces. In fact, the only $B$-scroll that is conoidal surface is the $B$-scroll over a generalized null cubic, i.e. a parabolic null cylinder. Examples of $B$-scrolls 
include flat $B$-scrolls, such as parabolic null cylinders, and non-flat totally umbilical ones, such as pseudo-spheres $S_{1}^{2}(p, r)$.

EXAmple 6.1. A pseudosphere $S_{1}^{2}(p, r)$ is a ruled surface whose both families of rulings are null. The following parametrization is the local parametrization of $S_{1}^{2}(0, r)$ as a $B$-scroll (Figure 1)

$$
f(u, v)=(r, 0,0)+\frac{1}{\sqrt{2}}(0, u, u)+v\left(-\frac{u}{r},-\frac{u^{2}}{2 \sqrt{2} r^{2}}+\frac{1}{\sqrt{2}},-\frac{u^{2}}{2 \sqrt{2} r^{2}}-\frac{1}{\sqrt{2}}\right) .
$$

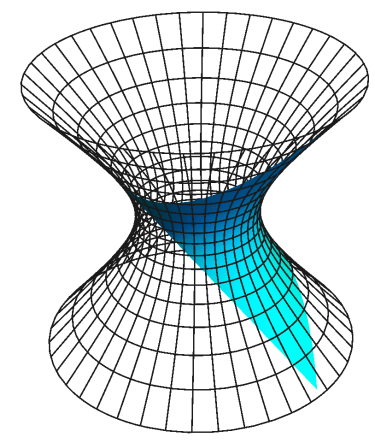

Figure 1. A pseudosphere $S_{1}^{2}(p, r)$ with a patch parametrized as a $B$-scroll

Now we consider Minding isometries of $B$-scrolls. From the first fundamental form (6.2) we see that $k_{3}$ is preserved (hence $K$ and $H$ as well), and $k_{2}$ can vary without affecting the first fundamental form.

TheOREM 6.2. A B-scroll $S\left(k_{2}, k_{3}\right)$ can be mapped by a Minding isometry into a $B$-scroll $\bar{S}\left(\overline{k_{2}}, k_{3}\right)$ so that:

1. the $B$-scroll $\bar{S}$ is a B-scroll of constant slope;

2. a given curve $v=v(u)$ on $S$ is mapped into an asymptotic curve of $\bar{S}$;

3. a given geodesic on $S$ is mapped into a straight line of $\bar{S}$;

4. a given curve $v=v(u)$ on $S$ is mapped into a plane curve on $\bar{S}$;

Proof. $\quad 1$. By varying $\bar{k}_{2}$ we obtain $B$-scroll of constant slope.

2. An asymptotic curve on $\overline{\mathbb{S}}$ satisfies

$$
\left(\bar{k}_{2}+v k_{3}^{\prime}+v^{2} k_{3}^{3}\right) \mathrm{d} u^{2}+2 k_{3} \mathrm{~d} u \mathrm{~d} v=0 .
$$

When $v=v(u)$ is given, $\bar{k}_{2}$ can be calculated. 
3. A curve $v=v(u)$ on $\mathbb{S}$ with parametrization $\alpha(u)=c(u)+v(u) B(u)$ is a straight line when $\alpha^{\prime}$ i $\alpha^{\prime \prime}$ are linearly dependent, i.e. when matrix

$$
\left[\begin{array}{ccc}
1 & v^{\prime} & v k_{3} \\
-v k_{3}^{2} & -k_{2} k_{3} v+v^{\prime \prime} & k_{2}+2 v^{\prime} k_{3}+v k_{3}^{\prime}
\end{array}\right]
$$

is of rank 1. From this we obtain equations

$$
\left|\begin{array}{cc}
1 & v^{\prime} \\
-v k_{3}^{2} & -k_{2} k_{3} v+v^{\prime \prime}
\end{array}\right|=0
$$

and

$$
\left|\begin{array}{cc}
1 & v^{\prime}+\left(v k_{3}\right)^{2} \\
-v k_{3}^{2} & -k_{2} k_{3} v+v^{\prime \prime}+v k_{3}\left(k_{2}+2 v^{\prime} k_{3}+v k_{3}^{\prime}\right)
\end{array}\right|=0 .
$$

Equation (6.7) is the differential equation of a geodesic on a $B$-scroll. Since $v=v(u)$ is geodesic, it fulfills equation (6.7) and from equation of the form (6.6) we obtain $\bar{k}_{2}$.

4. A curve $\alpha$ is a plane curve if and only if $\operatorname{det}\left(\alpha^{\prime}, \alpha^{\prime \prime}, \alpha^{\prime \prime \prime}\right)=0$. From this condition we obtain the equation for $\bar{k}_{2}$ of the following form

$$
G k_{2}^{\prime}+F_{1} k_{2}+F_{2} k_{2}^{2}+F_{3} k_{2}^{3}=0,
$$

where $G, F_{1}, F_{2}, F_{3}: I \rightarrow \mathbb{R}$ are known functions which depend on $v, k_{3}$ and their derivatives.

EXAMPLE 6.3. A null-ruled surface with $k_{3}=$ const. $\neq 0$ is called a isoparametric surface, [2]. These surfaces are surfaces of constant Gaussian and mean curvatures. If such a surface is a surface of constant slope, then, when parametrized as a $B$-scroll, we have $k_{2}=$ const. as well. The special case is $S_{1}^{2}(p, r)$. The general parametrization of such a surface is given by (see [2])

$$
f(u, v)=\left(g(u), 0,-a u+\int g^{\prime}(u) \cos u d u\right)+v(\cos u, \sin u, 1) .
$$

All these surfaces are locally isometric in Minding sense to $S_{1}^{2}(p, r)$ (Figure 2).

\section{Example: Helicoidal RUled SuRfaces}

A helicoidal ruled surface $S$ in $\mathbb{R}_{1}^{3}$ is the orbit of a straight line under a one-parameter group of helicoidal motions, i.e. a rotation followed by a translation in the direction of the axis of rotation. Rotations include all three 

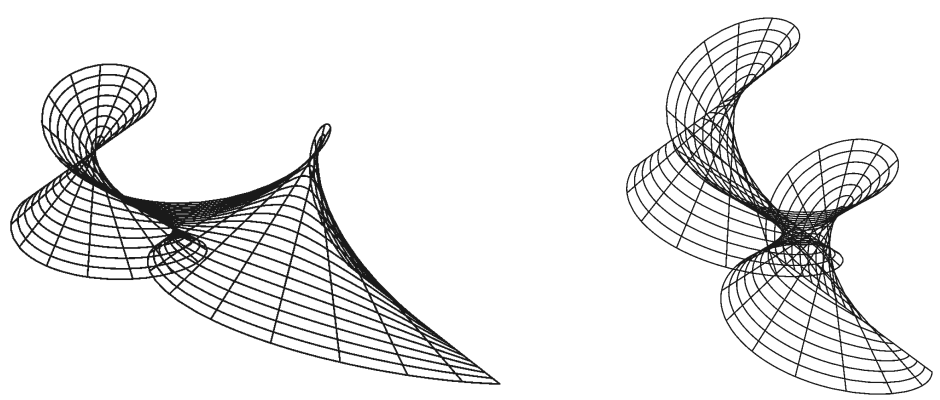

Figure 2. Examples of isoparametric surfaces

types of rotations, around a timelike, spacelike and null (lightlike) axis given by their canonical forms respectively

$$
\left(\begin{array}{ccc}
\cos u & \sin u & 0 \\
-\sin u & \cos u & 0 \\
0 & 0 & 1
\end{array}\right),\left(\begin{array}{ccc}
1 & 0 & 0 \\
0 & \cosh u & \sinh u \\
0 & \sinh u & \cosh u
\end{array}\right),\left(\begin{array}{ccc}
1-\frac{u^{2}}{2} & u & \frac{u^{2}}{2} \\
-u & 1 & u \\
-\frac{u^{2}}{2} & u & 1+\frac{u^{2}}{2}
\end{array}\right) \text {. }
$$

As we will show, these surface are ruled surfaces with constant curvature, torsion and striction. By Theorem 4.3, we can conclude that they are Minding images of ruled surfaces of same type, with the same curvature $\kappa=$ const. and striction $\sigma=$ const. and arbitrary torsion $\tau$.

Case 1. If we consider a rotation of a straight line around timelike axis, without loss of generality, we may assume that the line lies in the $y z$-plane (or plane parallel to the $y z$-plane). We consider a straight line with timelike, spacelike and null direction $e$ as $e=(0, \cosh \alpha, \sinh \alpha), e=(0, \sinh \alpha, \cosh \alpha)$, $e=(0,1,1)$, respectively.

For a spacelike direction $e$ (analogously in other cases), a helicoidal surface $S$ is parametrized as

$$
f(u, v)=\left(\begin{array}{ccc}
\cos u & \sin u & 0 \\
-\sin u & \cos u & 0 \\
0 & 0 & 1
\end{array}\right)\left(\begin{array}{c}
r \\
\cosh \alpha \cdot v \\
\sinh \alpha \cdot v
\end{array}\right)+\left(\begin{array}{c}
0 \\
0 \\
h \cdot u
\end{array}\right) .
$$

Therefore its parametrization as a ruled surface is given by

(7.1) $f(u, v)=(r \cos u,-r \sin u, h \cdot u)+v(\cosh \alpha \sin u, \cosh \alpha \cos u, \sinh \alpha)$.

This surface is a ruled surface of a type $S_{1}$, (resp. $S_{3}$ ) with the curvature, torsion and striction equal to (for surfaces of type $S_{1}$ )

$$
\kappa=\frac{\cosh \alpha}{\sqrt{r^{2}-h^{2}}}, \quad \tau=\frac{-\sinh \alpha}{\sqrt{r^{2}-h^{2}}}, \quad \sigma=\operatorname{arcsinh}\left(\frac{-r \cosh \alpha-h \sinh \alpha}{\sqrt{r^{2}-h^{2}}}\right) .
$$


Therefore, these surfaces are surfaces of constant slope, in particular, conoidal surfaces. In particular, a classical helicoid of the first kind is obtained for $\alpha=0, h=1, r=0$. A hyperboloid of one sheet is obtained as a rotational surface (for $h=0$ ) and its rulings can be either timelike, spacelike or null.

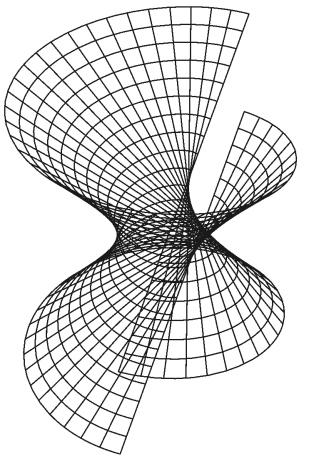

Figure 3. Helicoidal ruled surface with parametrization (7.1) for $r=2, h=1, \alpha=2$

Case 2. We consider rotations around a spacelike axis ( $x$-axis). A straight line can be taken either in a plane parallel to a spacelike $x y$-plane or in a plane parallel to a timelike $x z$-plane.

If a line lies in a plane parallel to the $x y$-plane and has a direction $e=(\cos \alpha, \sin \alpha, 0)$, then a surface $S$ is parametrized by

$$
f(u, v)=\left(\begin{array}{ccc}
1 & 0 & 0 \\
0 & \cosh u & \sinh u \\
0 & \sinh u & \cosh u
\end{array}\right)\left(\begin{array}{c}
\cos \alpha \cdot v \\
\sin \alpha \cdot v \\
r
\end{array}\right)+\left(\begin{array}{c}
h \cdot u \\
0 \\
0
\end{array}\right)
$$

that is,

(7.2) $f(u, v)=(h \cdot u, r \sinh u, r \cosh u)+v(\cos \alpha, \sin \alpha \cosh u, \sin \alpha \sinh u)$.

Under a helicoidal motion, a ruled surface of a type $S_{1}$ is obtained with

$$
\kappa=\frac{|\sin \alpha|}{\sqrt{r^{2}+h^{2}}}, \quad \tau=\frac{-\cos \alpha}{\sqrt{r^{2}+h^{2}}}, \quad \sigma=\operatorname{arccosh}\left(\frac{h \cos \alpha+r \sin \alpha}{\sqrt{r^{2}+h^{2}}}\right) .
$$

In particular, a helicoid of the second kind is obtained for $\alpha=\frac{\pi}{2}, h=1, r=0$.

Now, if we assume that a straight line lies in a plane parallel to the $x z$-plane, we can consider a straight line with spacelike, timelike and lightlike direction. For a timelike direction $e=(\sinh \alpha, 0, \cosh \alpha)$ a surface $S$ is 


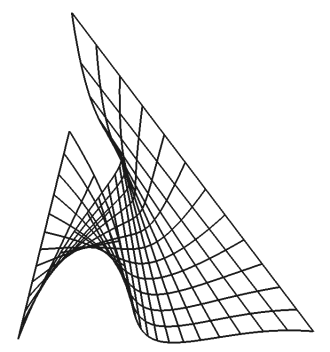

Figure 4. A helicoidal ruled surface with parametrization (7.2) for $r=1, h=1, \alpha=2.5$

parametrized as

$$
f(u, v)=\left(\begin{array}{ccc}
1 & 0 & 0 \\
0 & \cosh u & \sinh u \\
0 & \sinh u & \cosh u
\end{array}\right)\left(\begin{array}{c}
\sinh \alpha \cdot v \\
r \\
\cosh \alpha \cdot v
\end{array}\right)+\left(\begin{array}{c}
h \cdot u \\
0 \\
0
\end{array}\right),
$$

that is,

$$
f(u, v)=(h \cdot u, r \cosh u, r \sinh u)+v(\sinh \alpha, \cosh \alpha \sinh u, \cosh \alpha \cosh u) .
$$

By this motion, a ruled surface of a type $S_{2}$, (resp. $S_{3}$ ) is obtained with

$$
\kappa=\frac{\cosh \alpha}{\sqrt{h^{2}-r^{2}}}, \quad \tau=\frac{\sinh \alpha}{\sqrt{h^{2}-r^{2}}}, \quad \sigma=\operatorname{arccosh}\left(\frac{h \sinh \alpha-r \cosh \alpha}{\sqrt{h^{2}-r^{2}}}\right) .
$$

A classical helicoid of the third kind is obtained for $\alpha=0, h=1, r=0$.

Case 3. Finally, we consider helicoidal motions around the null $(x, 0, x)$-axis. We assume that a straight line lies in a plane parallel to $x=z$ plane. The obtained surface $S$ is parametrized by

$$
f(u, v)=\left(\begin{array}{ccc}
1-\frac{u^{2}}{2} & u & \frac{u^{2}}{2} \\
-u & 1 & u \\
-\frac{u^{2}}{2} & u & 1+\frac{u^{2}}{2}
\end{array}\right)\left(\begin{array}{c}
a \cdot v+b \\
c \cdot v \\
a \cdot v+b
\end{array}\right)+h\left(\begin{array}{c}
\frac{u^{3}}{3}-u \\
u^{2} \\
\frac{u^{3}}{3}+u
\end{array}\right),
$$

that is,

$$
f(u, v)=\left(b+h\left(\frac{u^{3}}{3}-u, h u^{2}, b+h\left(\frac{u^{3}}{3}+u\right)\right)+v(a+u, c, a+u) .\right.
$$

By this motion, we obtain a ruled surface with a null base curve and rulings that have either a spacelike, timelike or null direction. According to [10], the surfaces with null rulings can be reparametrized as $B$-scrolls, 


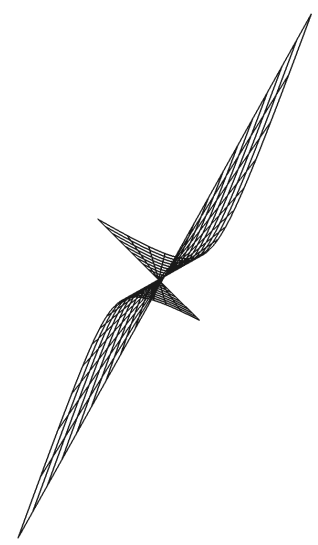

FiguRE 5. A helicoidal ruled surface with parametrization (7.3) for $r=1, h=2, \alpha=2.5$

while those with spacelike or timelike rulings can be reparametrized either as surfaces of class $M_{1}^{1}$ or of class $M_{1}^{0}$. Conjugate of Enneper's surface of second kind (Figure 6) with a parametrization

$$
f(u, v)=\left(\left(\frac{u^{3}}{3}-u\right)+u v, u^{2}+v,\left(\frac{u^{3}}{3}+u\right)+u v\right)
$$

is an example of a ruled surface obtained under a helicoidal motion of a line $\beta(v)=(v, v, v)$ around a null axis. Theorem 5.1 implies that there are no ruled surfaces that are locally isometric to this surface other than congruent one.

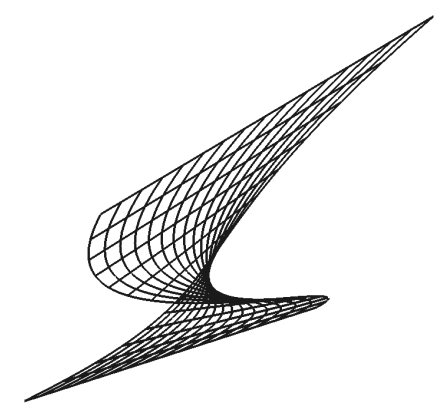

Figure 6. Conjugate of Enneper's surface of the second kind 


\section{REFERENCES}

[1] F. Dillen and W. Kühnel, Ruled Weingarten surfaces in Minkowski 3-space, Manuscripta Math. 98 (1999), 307-320.

[2] F. Dillen, I. Van de Woestyne, L. Verstraelen and J. Walrave, Ruled surfaces of finite type in 3-dimensional Minkowski space, Results Math. 27 (1995),250-255.

[3] F. Dillen and W. Sodsiri, Ruled surfaces of Weingarten type in Minkowski 3-space, J. Geom. 83 (2005), 10-21.

[4] B. Divjak and Ž. Milin Šipuš, Minding isometries of ruled surfaces in pseudo-Galilean space, J. Geom. 77 (2003), 35-47.

[5] L. P. Eisenhart, A Treatise of the Differential Geometry of Curves and Surfaces, The Cornell University Library Digital Collections, 1991/ Ginn \& co. 1909.

[6] S. Ersoy and M. Tosun, Lamarle formula in 3-dimensional Lorentz space, Math. Commun. 20 (2010), 1-15.

[7] G. Grantcharov and R. Salom, Bonnet pairs of surfaces in Minkowski space, Afr. Diaspora J. Math. (N.S.) 14 (2012), 57-75.

[8] E. Güler and A. T. Vanli, Bour's theorem in Minkowski 3-space, J. Math. Kyoto Univ. 46 (2006), 47-63.

[9] M. Hamann and B. Odehnal, Conchoidal ruled surfaces, in: Proceedings of the 15th ICGG, Montreal, 2012, pp. 262-268.

[10] J. Inoguchi and S. Lee, Null curves in Minkowski 3-space, Int. Electron. J. Geom. 1 (2008), 40-83.

[11] D. S. Kim, Y. H. Kim and D. W. Yoon, On ruled surfaces in Minkowski spaces, Commun. Korean Math. Soc 18 (2003), 289-295.

[12] Y. H. Kim and D. W. Yoon, Classification of ruled surfaces in Minkowski 3-spaces, J. Geom. Phys. 49 (2004), 89-100.

[13] Y. H. Kim and D. W. Yoon, On non-developable ruled surfaces in Lorentz-Minkowski 3-spaces, Taiwanese J. Math. 11 (2007), 197-214.

[14] E. Kruppa, Natürliche Geometrie der Mindingschen Verbiegungen der Strahlflächen, Monatsh. Math. 55 (1951), 340-345.

[15] R. Lopez, Differential geometry of curves and surfaces in Lorentz-Minkowski space, Int. Electron. J. Geom. 7 (2014), 44-107.

[16] F. Manhart, Bonnet-Thomsen surfaces in Minkowski geometry, J. Geom. 106 (2015), $47-61$.

[17] Ž. Milin Šipuš and B. Divjak, Mappings of ruled surfaces in simply isotropic space $I_{3}^{1}$ that preserve the generators, Monatsh. Math. 139 (2003), 235-245.

[18] Ž. Milin Šipuš and Lj. Primorac Gajčić, Ruled surfaces of constant slope in 3Minkowski space, in: Proceedings of the 16th ICGG, Innsbruck, 2014, pp. 1087-1094.

[19] Lj. Primorac Gajčić, Mappings of ruled surfaces in Minkowski space, Ph.D. Thesis, Department of Mathematics, University of Zagreb, Zagreb, 2016. 


\section{Mindingove izometrije pravčastih ploha $u$ Lorentz-Minkowskijevom prostoru}

\section{Ljiljana Primorac Gajčić i Željka Milin Šipuš}

SAŽETAK. U ovom radu proučavamo izometrije pravčastih ploha u Lorentz-Minkowskijevom prostoru koje čuvaju izvodnice. Posebna pažnja je posvećena klasama pravčastih ploha koje nemaju svoj analogon $\mathrm{u}$ euklidskom prostoru. Također dajemo primjere pravčastih ploha s određenim svojstvima koje su lokalno izometrične pri čemu su izvodnice očuvane.

Ljiljana Primorac Gajčić

Department of Mathematics

University of Osijek

31000 Osijek, Croatia

E-mail: ljiljana.primorac@mathos.hr

Željka Milin Šipuš

Faculty of Science, Department of Mathematics

University of Zagreb

10000 Zagreb, Croatia

E-mail: milin@math.hr

Received: 10.3.2018.

Revised: 27.9.2018.

Accepted: 22.10.2018. 